\title{
SOME PROPERTIES OF ENDOMORPHISMS IN RESIDUALLY FINITE GROUPS
}

\author{
RONALD HIRSHON \\ Received 11 March; revised 10 August 1976) \\ Communicated by M. F. Newman
}

\begin{abstract}
If $\varepsilon$ is an endomorphism of a finitely generated residually finite group onto a subgroup $F \varepsilon$ of finite index in $F$, then there exists a positive integer $k$ such that $\varepsilon$ is an isomorphism on $F \varepsilon^{k}$. If $K$ is the kernel of $\varepsilon$, then $K$ is a finite group so that if $F$ is a non trivial free product or if $F$ is torsion free, then $\varepsilon$ is an isomorphism on $F$. If $\varepsilon$ is an endomorphism of a finitely generated residually finite group onto a subgroup $F \varepsilon$ (not necessarily of finite index in $F$ ) and if the kernel of $\varepsilon$ obeys the minimal condition for subgroups, then there exists a positive integer $k$ such that $\varepsilon$ is an isomorphism on $F_{\varepsilon}{ }^{k}$.
\end{abstract}

\section{Introduction}

A pleasant property for a group to possess is that of being residually finite. Finitely generated residually finite groups are in fact quite abundant. The construction of B. H. Neumann [Kurosh, (1956), p. 51] shows that the set of non-isomorphic two-generator groups of this kind has the power of the continuum. For a survey of some relevant literature see Magnus (1969). In this paper, we present some information concerning the endomorphisms of these groups.

\section{Statement of results}

The fact that every surjective endomorphism of a finitely generated residually finite group is an automorphism [Magnus, Karrass, Solitar, (1966), p. 415] is a well known result. We will prove the following generalization:

Theorem 1: If $\alpha$ is an endomorphism of a finitely generated residually finite group $G$ onto a subgroup $G \alpha$ of finite index in $G$, then there exists a positive integer $k$ such that $\alpha$ is an isomorphism on $G \alpha^{k}$.

Note that if $K$ is the kernel of $\alpha$ this means $K \cap G \alpha^{k}=1$ so that elements in $K$ determine distinct right cosets of $G \alpha^{k}$. But $\left[G: G \alpha^{k}\right]$ is finite. Hence we see: 
Corollary 1: $K$ is a finite group.

Also since a non-trivial free product cannot have a finite normal subgroup we see:

Corollary 2: If $G=A * B, A \neq 1, B \neq 1$. then $\alpha$ is an isomorphism on $G$.

Also we may obviously state:

Corollary 3. If $G$ is torsion free, $\alpha$ is an isomorphism.

The hypothesis that $G$ is finitely generated is clearly necessary, for in infinitely generated groups the situation is quite different. For example, if $G$ is the direct product of a countable number of copies of a finite group $K$ say

$$
G=K_{1} \times K_{2} \times \cdots, \quad K_{i} \approx K, K \neq 1
$$

and if we define $\alpha$ by

$$
\left(k_{1}, k_{2}, k_{3}, \cdots\right) \alpha=\left(k_{2}, k_{3}, \cdots\right)
$$

then $\alpha$ is not an isomorphism on $G \alpha^{k}$ for any $k$.

We have not been able to answer the following question: If $\alpha$ is an endomorphism of a finitely generated residually finite group $G$, does there exist a $k$ such that $\alpha$ is an isomorphism on $G \alpha^{k}$ ?

However we will prove Theorems 2 and 3 stated below, Theorem 2 being a partial converse of Corollary 1 .

THEOREM 2. If $\alpha$ is an endomorphism of a finitely generated residually finite group $G$ and the kernel of $\alpha$ obeys the minimal condition for subgroups then there exists a positive integer $k$ such that $\alpha$ is an isomorphism on $G \alpha^{k}$.

Our previous example again shows the necessity that $G$ be finitely generated here.

THEOREM 3. Let $\alpha$ be an endomorphism of the finitely generated residually finite group $G$. Let $K_{i}$ denote the kernel of $\alpha^{i}$. If $r$ is any integer $r \geqq 0$, a necessary and sufficient condition that $\alpha$ be an isomorphism on $G \alpha^{\prime}$ is that $\alpha$ map the union of the groups $K_{i} \cap G \alpha^{\prime}, i=1,2,3 \cdots$ onto itself.

\section{The proof of Theorems 1-3}

Lemma 1. Let $G$ be a finitely generated residually finite group and let $G=G_{1}, G_{2}, \cdots$ be a sequence of subgroups of $G$ with $G_{i+1} \subset G_{i}$ for $i=1,2, \cdots$. Let $\theta_{i}$ be an endomorphism of $G_{i}$ such that $G_{i} \theta_{i}=G_{i+1}$. Let the kernel of $\theta_{i}$ be $K^{i}$. Then the intersection of the $K^{i}$ is precisely the identity element. 
To prove the above we will show that the intersection of the $K^{i}$ is contained in any normal subgroup $N$ of finite index in $G$ and must consequently be 1. Let $N_{i}=G_{i} \cap N$. Let $L_{i}$ be the pre-image of $N_{i}$ under $\alpha_{i}$ where $\alpha_{i}$ is the composite of $\theta_{1}, \theta_{2}, \cdots, \theta_{i-1}$. Hence

$$
G / L_{\mathrm{i}} \approx G_{\mathrm{i}} / G_{\mathrm{i}} \cap N
$$

so that infinitely many of the $L_{\mathrm{i}}$ are equal [Magnus, Karrass, Solitar, exercise 19. p. 102, (1966)]. Also if $L_{i}=L_{j}(i<j)$ and if $\beta_{i, j}$ is the composite of $\theta_{i}, \theta_{t-1} \cdots, \theta_{i-1}$ then $N_{i} \beta_{i, j}=N_{i}$ and $\beta_{i, j}$ induces a homomorphism of $G_{i} / N_{i}$ onto $G_{i} / N_{i}$. Since the groups $G_{i} / N_{i}$ are of bounded order we can choose $i$ and $j$ large enough so that this homomorphism is an isomorphism. This implies in particular that $K^{i} \subset N_{i} \subset N$.

Now for the proof of Theorem 1, let $G_{i}=G \alpha^{i-1}$ and let $\theta_{i}$ denote the restriction of $\alpha$ to $G_{i}$. Let $K^{i}=K \cap G_{i}, i \geqq 1$, where $K$ is the kernel of $\alpha$. Let $N_{i}$ be the intersection of the conjugates $g^{-1} G_{i+1} g, g \in G_{i}$ so that $N_{i}$ is a normal subgroup of finite index of $G_{i}$. Since $G_{i} \theta_{i}=G_{i+1}$ and $N_{i} \theta_{i}=N_{i+1}, \theta_{i}$ induces a homomorphisms $\bar{\theta}_{i}$ of $G_{i} / N_{i}$ onto $G_{i+1} / N_{i+1}$. Since all the above quotient groups are finite, ultimately say for $i \geqq p, \bar{\theta}_{\mathrm{i}}$ is an isomorphism. In particular for $i \geqq p$ we have,

$$
K \cap G_{i} \subset N_{i} \cap G_{i+1}
$$

Since $G_{i+1} \subset G_{i}$ this means that for $i \geqq p$

$$
K^{i+1}=K \cap G_{i+1}=K^{i}
$$

Hence the intersection of the $K^{i}$ is $K^{p}$. By Lemma $1, K^{p}=1$. Hence $\alpha$ is an isomorphism on $G \alpha^{p-1}$.

For the proof of Theorem 2, the groups $K \cap G_{i}=K^{i}$ form a descending sequence of subgroups of $K$ so that ultimately the $K^{i}$ are identical and consequently ultimately $K^{i}=1$, by Lemma 1 .

Since each $G \alpha^{i}$ is a finitely generated residually finite group, to prove Theorem 3 it suffices to consider only the case $r=0$. If $\alpha$ is an isomorphism on $G$ then each $K_{i}=1$ so that $\alpha$ maps the union of the $K_{i}$ onto itself. Conversely let $L$ be the union of the $K_{i}$ and suppose that $L \alpha=L$. Then $L$ is contained in the intersection of the $G \alpha^{i}$. In particular $K$ is contained in the intersection of the $G \alpha^{\prime}$ so $K$ is in the intersection of the $K^{i}$. Hence $K=1$, again by Lemma 1 .

\section{Acknowledgement}

The author thanks the referee for several useful suggestions. 


\section{References}

A. G. Kurosh (1956), The Theory of Groups, Vol. 2 (translated by K. Hirsch from the second Russian edition). Chelsea, New York.

W. Magnus, A. Karrass and D. Solitar (1966), Combinatorial Group Theory (John Wiley and Sons, New York).

W. Magnus (1969), 'Residually finite groups', Bull. Amer. Math Soc, 75, 305-316.

Department of Mathematics,

Polytechnic Institute of New York,

Brooklyn, New York,

U.S.A. 